\title{
Correction to: Long-acting bronchodilators improve exercise capacity in COPD patients: a systematic review and meta-analysis
}

Fabiano Di Marco ${ }^{1 *}$, Giovanni Sotgiu², Pierachille Santus ${ }^{3,4}$, Denis E. O'Donnell ${ }^{5}$, Kai-Michael Beeh ${ }^{6}$, Simone Dore ${ }^{2}$, Maria Adelaide Roggi ${ }^{7}$, Lisa Giuliani ${ }^{1}$, Francesco Blasi ${ }^{8,9}$ and Stefano Centanni ${ }^{1}$

\section{Correction}

In the original publication [1] is an incorrect sentence in the abstract under Conclusions. The modified text is indicated in bold. The correct version can be found in this Erratum:

Incorrect version:

Conclusions: Long-acting bronchodilators improve exercise capacity in COPD. The main effect of long-acting bronchodilators seems to be a decrease of basal IC rather than a modification of dynamic hyperinflation during exercise. The efficacy in terms of endurance time seems higher in studies which enrolled patients with hyperinflation, with a similar efficacy on walking or cycling.

Correct version

Conclusions: Long-acting bronchodilators improve exercise capacity in COPD. The main effect of long-acting bronchodilators seems to be an increase of basal IC rather than a modification of dynamic hyperinflation during exercise. The efficacy in terms of endurance time seems higher in studies which enrolled patients with hyperinflation, with a similar efficacy on walking or cycling.

\footnotetext{
Author details

${ }^{1}$ Respiratory Unit, Ospedale San Paolo, Department of Health Science, Università degli Studi di Milano, Via A. di Rudinì, 8-20142 Milan, Italy. ${ }^{2}$ Clinical Epidemiology and Medical Statistics Unit, Department of Biomedical Sciences, University of Sassari, Sassari, Italy. ${ }^{3}$ Department of Biomedical And Clinical Sciences (DIBIC), University of Milan, Milan, Italy. ${ }^{4}$ Respiratory Unit, "Luigi Sacco" University Hospital; ASST Fatebenefratelli-Sacco, Milan, Italy. ${ }^{5}$ Division of Respiratory and Critical Care Medicine, Respiratory Investigation Unit, Queen's University and Kingston General Hospital, Kingston, ON, Canada. ${ }^{6}$ Insaf Respiratory Research Institute, Wiesbaden, Germany. ${ }^{7}$ Cardiothoracic and Vascular Department, University of Pisa, Pisa, Italy. ${ }^{8}$ Department of Pathophysiology and Transplantation, University of Milan, Milan, Italy. ${ }^{9}$ Internal Medicine Department, Respiratory Unit and Cystic
}

Fibrosis Adult Center, Fondazione IRCCS Ca' Granda Ospedale Maggiore Policlinic, Milan, Italy.

Published online: 24 April 2018

\section{Reference}

1. Di Marco F, et al. Long-acting bronchodilators improve exercise capacity in COPD patients: a systematic review and meta-analysis. Respir Res. 2018;19:18. https://doi.org/10.1186/s12931-018-0721-3

* Correspondence: fabiano.dimarco@unimi.it

${ }^{1}$ Respiratory Unit, Ospedale San Paolo, Department of Health Science,

Università degli Studi di Milano, Via A. di Rudinì, 8-20142 Milan, Italy 